\title{
Power Allocation for Reliable SIC Detection of Rectangular QAM-based NOMA Systems
}

\author{
Youssef Iraqi, Senior Member, IEEE, and Arafat Al-Dweik, Senior Member, IEEE
}

\begin{abstract}
This paper considers the power allocation for nonorthogonal multiple access (NOMA) users to enable using the successive interference cancellation (SIC) while providing reliable error performance. The derived closed-form expressions are applicable for arbitrary number of NOMA users each of which has a square or rectangular quadrature amplitude modulation (QAM) constellation with arbitrary order. The obtained numerical results show that power assignment process at the transmitter for the superposition process and at the receiver for the SIC process should be performed meticulously because the power difference between the weakest and strongest users can be tremendous when the number of users or the modulation orders increase. Moreover, the derived expressions can be used to reduce the computational complexity that is required to obtain the optimal power coefficients using brute force methods by significantly reducing the search space.
\end{abstract}

Index Terms-non-orthogonal multiple access (NOMA), successive interference cancellation (SIC), quadrature amplitude modulation (QAM), error floors, power assignment.

\section{INTRODUCTION}

D UE to the continuous demand for wireless services, significant efforts are currently devoted to design spectrally efficient multiple access (MAC) protocols. In particular, nonorthogonal multiple access (NOMA) has attracted significant attention due to its potential to serve more users using the same spectrum used by orthogonal MAC protocols. Nevertheless, NOMA generally has some challenges that should be resolved to achieve its full potential. Some of these challenges are the inter-user-interference (IUI), receiver complexity, and the requirement for meticulous power control [1].

In ${ }^{\left[R_{3,1}\right]}$ downlink power-domain NOMA, the users' symbols are multiplexed at the base station in the power domain by assigning high power to users with poor channel conditions, and low power to users with good channel conditions. Therefore, without loss of generality, a NOMA system that serves $N$ users whose channels' gains are $\alpha_{1}<\alpha_{2}<\cdots<\alpha_{N}$ allocates the powers such that $P_{1}>P_{2}>\cdots>P_{N}$. Consequently, the unique power assigned for each user enables the receiver to separate the data for each user using the maximum likelihood detector (MLD) [1], [2]. However, to use the less complex successive interference cancellation (SIC) detector, the power ordering is not sufficient to guarantee that symbols of all users will be detected reliably [3]. It is

Y. Iraqi and A. Al-Dweik are with the Department of Electrical Engineering and Computer Science, Khalifa University, Abu Dhabi, UAE. (e-mail: \{Youssef.Iraqi,Arafat.Dweik\}@ku.ac.ae).

A. Al-Dweik is also with the department of Electrical and Computer Engineering, Western University, London, ON, Canada. (e-mail: dweik@ fulbrightmail.org). worth noting that both the MLD and SIC detectors will have identical bit error rate (BER) given that the power assignment is performed such that the constellation for each user does not overlap with the constellations of other users [1].

Because the data for all users is multiplexed in the power domain, the power assignment for each user is a critical requirement for NOMA systems to offer reliable BER [1], [2], [4]-[8]. Generally speaking, the power assignment in the literature is performed to minimize the average BER of all users [4], or to satisfy the BER requirements for each user [1], [9]. Alternatively, the power can be assigned just to avoid the constellation overlapping for all users [2], [3], [10]-[12]. While [2], [11], [12] derived the power ranges for the case of two-user NOMA using quadrature amplitude modulation (QAM) with arbitrary order, Aldababsa et. al. [13] derived the power assignment ranges for an arbitrary number of users each of which uses binary phase shift keying (BPSK). More recently, Kara and Kaya [3] derived the ranges for the more general case that considers an arbitrary number of users and QAM with arbitrary modulation order. However, the derived formulas are valid only for square QAM constellations.

${ }^{\left[R_{2,2}\right]}$ Although the square QAM is widely used in practical applications, the rectangular QAM is commonly preferred for transmitting odd number of bits per symbol, because its power efficiency is significantly higher than phase shift keying (PSK), particularly for high modulation orders [14]. Therefore, the rectangular QAM has been also adopted for various applications such as digital video broadcasting (DVB) and digital multi-program television networks [14], [15]. Moreover, the low complexity modulation and demodulation made it attractive for high rate transmissions [16].

Based on the aforementioned literature, and the references listed therein, it can be noted that computing the power ranges for NOMA systems with an arbitrary number of users and a rectangular QAM with arbitrary modulation order remains an open research problem. Therefore, ${ }^{\left[R_{3,2}\right]}\left[R_{1,3}\right]$ the contribution of this correspondence can be summarized as:

- Derived the power ranges for downlink NOMA systems to enable the SIC receiver to work without constellation points overlap, and thus, avoid severe performance degradation.

- Using a generalized representation of constellation points for rectangular QAM, efficient closed-form expressions are recursively derived for NOMA systems with arbitrary number of users where each of which uses QAM with an arbitrary modulation order. Consequently, the work reported in the existing literature can be considered as special cases of the results presented in this work. 
- The derived expressions are further simplified for several cases of interest.

- The relation between power ranges and optimum power allocation is demonstrated graphically for the three users scenario.

- The obtained results are used to provide interesting insights about the power allocation for NOMA when the number of users or the modulation are large.

\section{SYSTEM MODEL}

Consider ${ }^{\left[R_{3,1}\right]}$ a downlink NOMA system with $N$ users each of which is using a rectangular QAM with modulation order $M_{i}=2^{k_{i}+1}, i \in\{1,2, \ldots, N\}$ and the integer $k_{i} \geq 1$. The superposition symbol transmitted by the base station can be written as

$$
X=\sum_{i=1}^{N} \beta_{i} x^{(i)}, x \in \mathbb{X}^{(i)} \text { and } X \in \mathbb{X}
$$

where $\beta_{i}^{2} \triangleq P_{i}$ is the power allocation coefficient for the $i$ th user, $x^{(i)}$ is the baseband modulated symbol of the $i$ th user with modulation order $M_{i}$ and set of constellation points $\mathbb{X}^{(i)}$. The modulation order of symbol $X$ is $\mathcal{M}=M_{1} \times M_{2} \times \cdots \times$ $M_{N}$ and the set of constellation points is $\mathbb{X}$.

The power coefficients are typically assigned subject to,

$$
\begin{gathered}
\sum_{i=1}^{N} P_{i}=1 \\
\beta_{i}>\beta_{i+1}>0, \forall i \in\{1, \ldots, \bar{N}\}
\end{gathered}
$$

where $\bar{N}=N-1$. Such assignment is necessary to provide fairness among different users by assigning far users more power. Therefore, (2a) and (2b) should be always satisfied throughout this article, even if they are not stated explicitly. However, these constraints are not sufficient to guarantee that the NOMA system will offer a reliable error performance using SIC detector. The justification is that the SIC detector is designed to use a single user detector successively. Therefore, the constellation for any user should not cross certain limits in the $x-y$ plane [3]. ${ }^{\left[R_{1,8}\right]}$ Fig. 1 shows two examples for constellation points overlap even when (2a) and (2b) are satisfied. The solid circles in the figure represent the cases where $P_{1} \gg P_{2}$, and thus, there is no constellation points overlap. The other constellation points are for the cases where $P_{1}>P_{2}$, but there is constellation points overlap. The constellation points that are supposed to be in the same quadrant are given the same shape. The overlap regions in both figures are shown as the shaded rectangles.

\section{A. Coordinates of Rectangular QAM}

For a rectangular QAM with modulation order $M$, there are $M / 4$ constellation points in each quadrant of the $x-y$ plane. If we define

$$
D_{1}=2^{\left\lceil\frac{1}{2} \log _{2}(M / 4)\right\rceil}
$$

and

$$
D_{2}=2^{\left\lfloor\frac{1}{2} \log _{2}(M / 4)\right\rfloor}
$$

$\left[R_{3,3}\right]\left[R_{1,5}\right]$ then the constellation points should be arranged over a rectangular $D_{1} \times D_{2}$ or $D_{2} \times D_{1}$ grid. In other words, if the constellation points in a quadrant are seen as points in a rectangular grid, $D_{1}$ will be the number of points on the side of the grid along the $x$-axis, and $D_{2}$ will be the number of points on the side of the grid along the $y$-axis. For the special case of a square QAM, we have $D_{1}=D_{2}$. As an example, if we consider $16-\mathrm{QAM}$, in each quadrant there are $2 \times 2$ constellation points. In this case, $D_{1}=D_{2}=2$. In the case of 32-QAM, the constellation points in each quadrant can be arranged as a grid of $4 \times 2$ points. In this case, $D_{1}=4$ and $D_{2}=2$. Note that 32 -QAM can also be arranged as a $2 \times 4$ grid in each quadrant. In this case $D_{1}=2$ and $D_{2}=4$.

The binary information bits are mapped into $M$ two-tuples $\left(x_{m}, y_{n}\right)$, each forming the transmitted symbol $s_{m, n}$. When the points are arranged over the rectangle $D_{1} \times D_{2}$, the coordinates of the constellation point $s_{m, n}$ are given by,

$$
\left(x_{m}, y_{n}\right)=\left(\frac{2 m-2 D_{1}-1}{\Delta}, \frac{2 n-2 D_{2}-1}{\Delta}\right)
$$

where $m \in\left[1, \ldots, 2 D_{1}\right], n \in\left[1, \ldots, 2 D_{2}\right]$, and $\Delta$ is a normalization factor chosen such that the average power of all symbols is unity,

$$
\Delta=\sqrt{\frac{1}{M} \sum_{m} \sum_{n}\left[2 m-2 D_{1}-1\right]^{2}+\left[2 n-2 D_{2}-1\right]^{2}} .
$$

When the points are arranged over the rectangle $D_{2} \times D_{1}$, the coordinates can be found using (5) and (6) while swapping $D_{1}$ and $D_{2}$.

Without loss of generality, let the shape of the rectangle in Quadrant I be $D_{x} \times D_{y}$, thus, the coordinates of the constellation points are

$$
\left(x_{m}, y_{n}\right)=\left(\frac{2 m-2 D_{x}-1}{\Delta}, \frac{2 n-2 D_{y}-1}{\Delta}\right)
$$

and

$$
\Delta=\sqrt{\frac{1}{M} \sum_{m} \sum_{n}\left[2 m-2 D_{x}-1\right]^{2}+\left[2 n-2 D_{y}-1\right]^{2}} .
$$

After some straightforward manipulations,

$$
\Delta=\sqrt{\frac{8}{3 M} D_{x} D_{y}\left[2 D_{x}^{2}+2 D_{y}^{2}-1\right]} .
$$

For a square QAM, $D_{x}=D_{y}$, and thus (9) becomes

$$
\Delta=\sqrt{\frac{2}{3}(M-1)} .
$$

\section{B. NOMA Power Allocation Basic Concept}

$\left[R_{2,3}\right]\left[R_{1,8}\right]$ Fig. 1 shows examples for two NOMA configurations where $N=2$. In both cases $M_{1}=4$, while $M_{2}=8$ in a) and 16 in b). To prevent constellations overlap, the closest points to the $x$ and/or $y$-axis should not cross the $x$ and/or $y$-axes. This point will be termed as the critical point, and it is represented by the solid circle which is marked by the arrow in Quadrant I. Although there are four critical points 


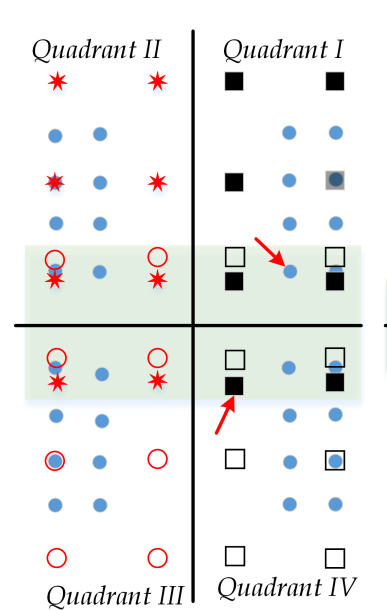

a)

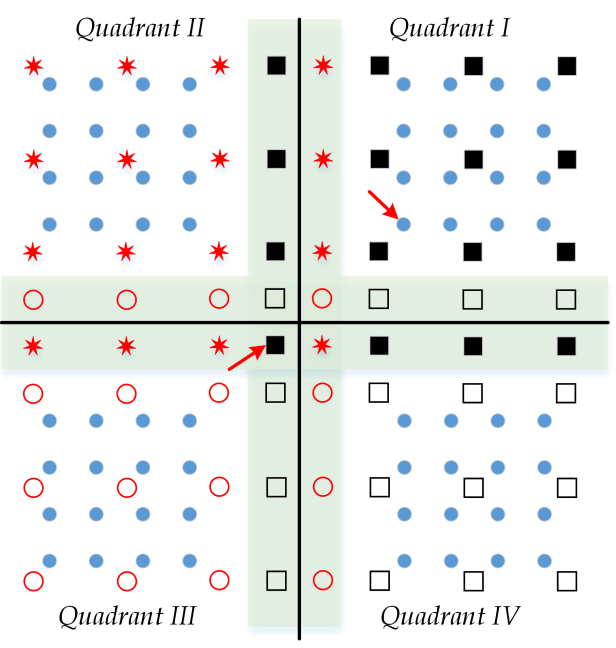

b)
Figure 1: Example of constellation points overlap for $N=2$ and $M_{1}=4$. a) $M_{2}=8, P_{1}=0.9,0.51$. b) $M_{2}=16, P_{1}=0.9,0.6$.

for this configuration, it is sufficient to consider only the point in Quadrant I due to the constellation symmetry about the $x$ and $y$-axis. Therefore, the power conditions can be calculated based on the critical point. Another example for $N=3$ is depicted in Fig. 2 where $M_{1}=M_{2}=M_{3}=4$. As can be noted from this figure, because $N=3$, there are two critical points in Quadrant I, marked by white circles in the solid squares. Therefore, to prevent overlap, the critical point that is close to the $x-y$ axis should not cross the $x-y$ axis, and the second point should not cross the virtual $x-y$ axis marked by the dashed lines.

$\left[R_{2,3}\right]\left[R_{3,3}\right]\left[R_{1,7}\right]$ The power allocation constraints are derived from the fact that the critical point should not cross the $x-y$ axis. For this we need to compute the coordinates of the critical point. These coordinates can be computed from the coordinates of specific constellation points of the involved users. For user 1 , the point of interest is the bottom-left constellation point in Quadrant I. For all the other users, the point of interest is the bottom-left constellation point in Quadrant III. For example, for a three-user NOMA using $M_{1}=M_{2}=M_{3}=4$, the coordinates of the critical point are $\beta_{1} s_{2,2}^{(1)}+\beta_{2} s_{1,1}^{(2)}+\beta_{3} s_{1,1}^{(3)}$, where $s_{m, n}^{(k)}$ are the coordinates of the constellation point $s_{m, n}$ of user $k$ as shown in Fig. 3 for the QPSK case. And for a three-user NOMA using $M_{1}=M_{2}=M_{3}=16$, the coordinates of the critical point are $\beta_{1} s_{3,3}^{(1)}+\beta_{2} s_{1,1}^{(2)}+\beta_{3} s_{1,1}^{(3)}$, where $s_{m, n}^{(k)}$ are the coordinates of the constellation point $s_{m, n}$ of user $k$ for the 16-QAM case.

Due to the nature of rectangular QAM, the constellation points are not necessarily symmetric about the diagonals in the $x-y$ plane. Therefore, the power constraints will be involving the $x$ - and $y$-coordinates of the critical point. More specifically, for user 1, the point of interest is in Quadrant I where $m=$ $D_{x}+1$ and $n=D_{y}+1$. The coordinates of the point of

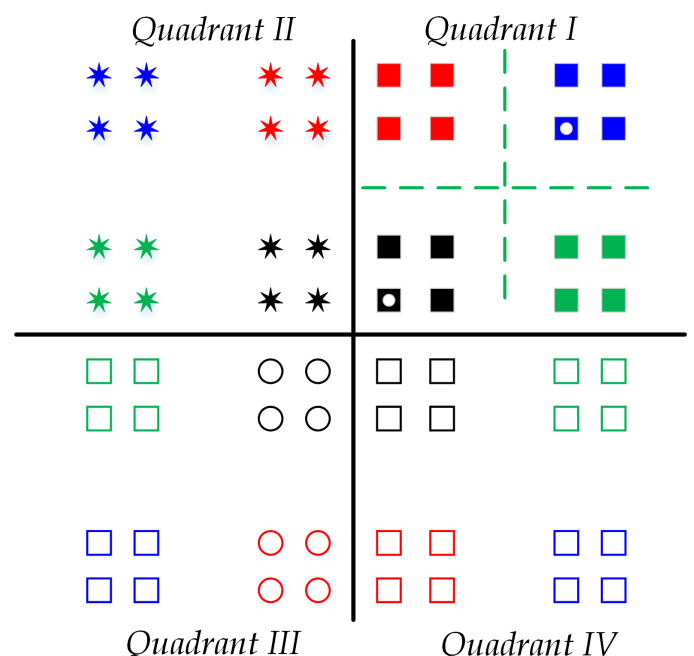

Figure 2: Constellation diagram of a three-user NOMA using $M_{1}=M_{2}=M_{3}=4$ showing various constellation points boundaries, $P_{1}=0.73, P_{2}=0.25$, and $P_{3}=0.02$.

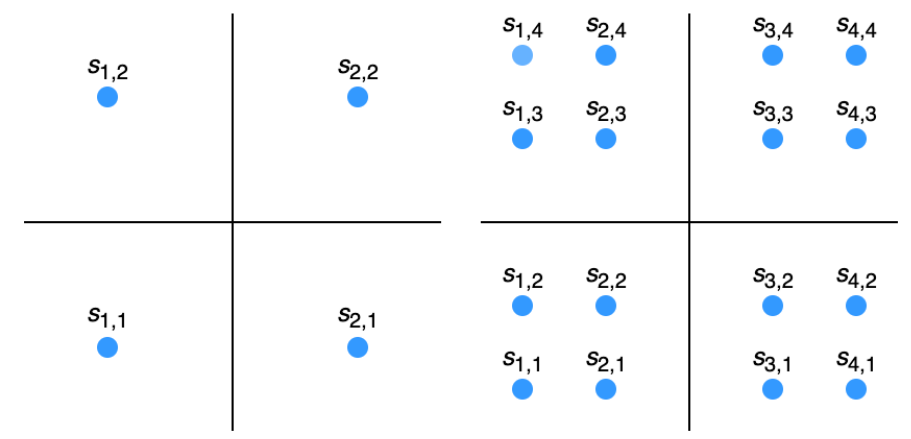

Figure 3: Labeled constellation points for QPSK and 16-QAM.

interest according to (7) are,

$$
\left(x_{D_{x}+1}, y_{D_{y}+1}\right)=\left(\frac{1}{\Delta}, \frac{1}{\Delta}\right) .
$$

The point of interest for the other users is the bottom-left constellation point in Quadrant III where $m=1$ and $n=1$. The coordinates of the point of interest according to (7) are

$$
\left(x_{1}, y_{1}\right)=\left(\frac{1-2 D_{x}}{\Delta}, \frac{1-2 D_{y}}{\Delta}\right) .
$$

Finally, the conditions should be applied recursively to enable assigning powers for the remaining users appropriately.

\section{Power Allocation Conditions}

Consider a NOMA system with $N$ users $\left\{U_{1}, U_{2}, \ldots, U_{N}\right\}$, modulation orders $\left\{M_{1}, M_{2}, \ldots, M_{N}\right\}$, shapes $\left\{\left(D_{x}^{(1)} \times\right.\right.$ $\left.\left.D_{y}^{(1)}\right),\left(D_{x}^{(2)} \times D_{y}^{(2)}\right), \ldots,\left(D_{x}^{(N)} \times D_{y}^{(N)}\right)\right\}$, and normalizing parameters $\left\{\Delta_{1}, \Delta_{2}, \ldots, \Delta_{N}\right\}$. Given that all users adopt a rectangular QAM constellation, then the power assignment for all users should satisfy the conditions given in the following proposition: 


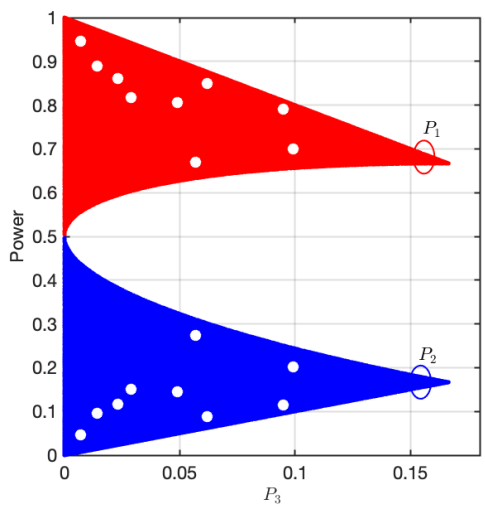

(a) $M=\{4,4,4\}$

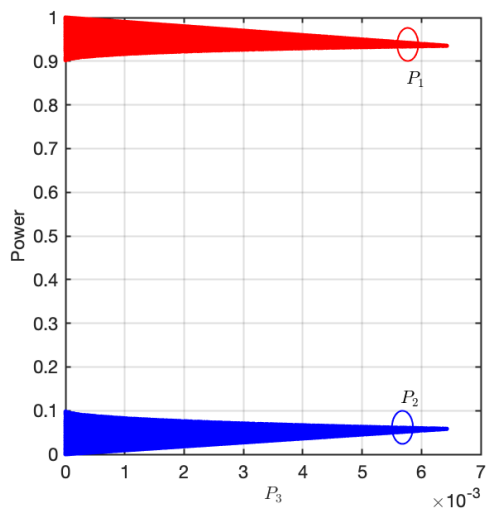

(b) $M=\{16,16,16\}$

Figure 4: Space of possible power allocation for $N=3$ and different values of $M_{i}$

Proposition 1. ${ }^{\left[R_{2,3}\right]}$ The power assignment for a NOMA system with $N$ users each of which is using a rectangular QAM with order $M_{i}, i=1,2, \ldots, N$ should satisfy the following conditions:

$$
\begin{gathered}
\beta_{u}>\Delta_{u} \max \left(\sum_{i=u+1}^{N} \frac{\beta_{i}}{\Delta_{i}}\left[2 D_{x}^{(i)}-1\right],\right. \\
\left.\sum_{i=u+1}^{N} \frac{\beta_{i}}{\Delta_{i}}\left[2 D_{y}^{(i)}-1\right]\right), u=1,2, \ldots, \bar{N} \\
\beta_{N}>0 \\
\sum_{i=1}^{N} \beta_{i}^{2}=1 .
\end{gathered}
$$

Proof. The condition for $U_{1}$ is:

$$
\frac{\beta_{1}}{\Delta_{1}}>0 \text {. }
$$

The conditions for $U_{2}$ are

$$
\begin{aligned}
& \frac{\beta_{1}}{\Delta_{1}}+\frac{\beta_{2}}{\Delta_{2}}\left[1-2 D_{x}^{(2)}\right]>0 \\
& \frac{\beta_{1}}{\Delta_{1}}+\frac{\beta_{2}}{\Delta_{2}}\left[1-2 D_{y}^{(2)}\right]>0
\end{aligned}
$$

and so forth. Therefore, the conditions considering the $N$ users are given by,

$$
\begin{gathered}
\frac{\beta_{1}}{\Delta_{1}}>0 \\
\frac{\beta_{1}}{\Delta_{1}}+\sum_{i=2}^{u} \frac{\beta_{i}}{\Delta_{i}}\left[1-2 D_{x}^{(i)}\right]>0 \forall u \in\{2,3, \ldots, N\} \\
\frac{\beta_{1}}{\Delta_{1}}+\sum_{i=2}^{u} \frac{\beta_{i}}{\Delta_{i}}\left[1-2 D_{y}^{(i)}\right]>0, \forall u \in\{2,3, \ldots, N\} .
\end{gathered}
$$

However, since the coordinates of the bottom-left constellation point in Quadrant III are negative, i.e., $(1-2 D) / \Delta<0$, then these conditions can be simplified to,

$$
\begin{aligned}
& \frac{\beta_{1}}{\Delta_{1}}+\sum_{i=2}^{N} \frac{\beta_{i}}{\Delta_{i}}\left[1-2 D_{x}^{(i)}\right]>0 \\
& \frac{\beta_{1}}{\Delta_{1}}+\sum_{i=2}^{N} \frac{\beta_{i}}{\Delta_{i}}\left[1-2 D_{y}^{(i)}\right]>0 .
\end{aligned}
$$

$\left[R_{1,7}\right]$ These are the conditions on the coordinates of the critical point from the perspective of $U_{1}$ that have to be positive, i.e., the critical point not crossing the $x$ or $y$-axes. Furthermore, these conditions have to be satisfied recursively for a system without user 1 and so on. Hence, the conditions for an $M$-ary rectangular QAM system with $N$ users can be summarized as:

$$
\begin{gathered}
\frac{\beta_{s}}{\Delta_{s}}+\sum_{i=s+1}^{N} \frac{\beta_{i}}{\Delta_{i}}\left[1-2 D_{x}^{(i)}\right]>0, \forall s \in\{1, \ldots, \bar{N}\} \\
\frac{\beta_{s}}{\Delta_{s}}+\sum_{i=s+1}^{N} \frac{\beta_{i}}{\Delta_{i}}\left[1-2 D_{y}^{(i)}\right]>0, \forall s \in\{1, \ldots, \bar{N}\} \\
\frac{\beta_{N}}{\Delta_{N}}>0 .
\end{gathered}
$$

These conditions can be rewritten as (13a) and (13b).

For the case that all users use square QAM, $D_{1}=D_{2}=D$ and the conditions (13a) and (13b) are simplified to

$$
\begin{gathered}
\beta_{u}>\Delta_{u} \sum_{i=u+1}^{N} \frac{\beta_{i}}{\Delta_{i}}\left[2 D^{(i)}-1\right], 1 \leq u \leq \bar{N} \\
\beta_{N}>0 .
\end{gathered}
$$

For this case, the conditions are similar to those in $[3$, Eq. 7]. Moreover, if $M_{i}=M \forall i$, then the conditions become

$$
\beta_{u}>[2 D-1] \sum_{\substack{i=u+1 \\ \beta_{N}>0 .}}^{N} \beta_{i}, 1 \leq u \leq \bar{N}
$$

As an example, if $M=4$ then $D=1$ and the conditions (13a) and (13b) become

$$
\begin{gathered}
\beta_{u}>\sum_{i=u+1}^{N} \beta_{i}, \quad 1 \leq u \leq \bar{N} \\
\beta_{N}>0 .
\end{gathered}
$$




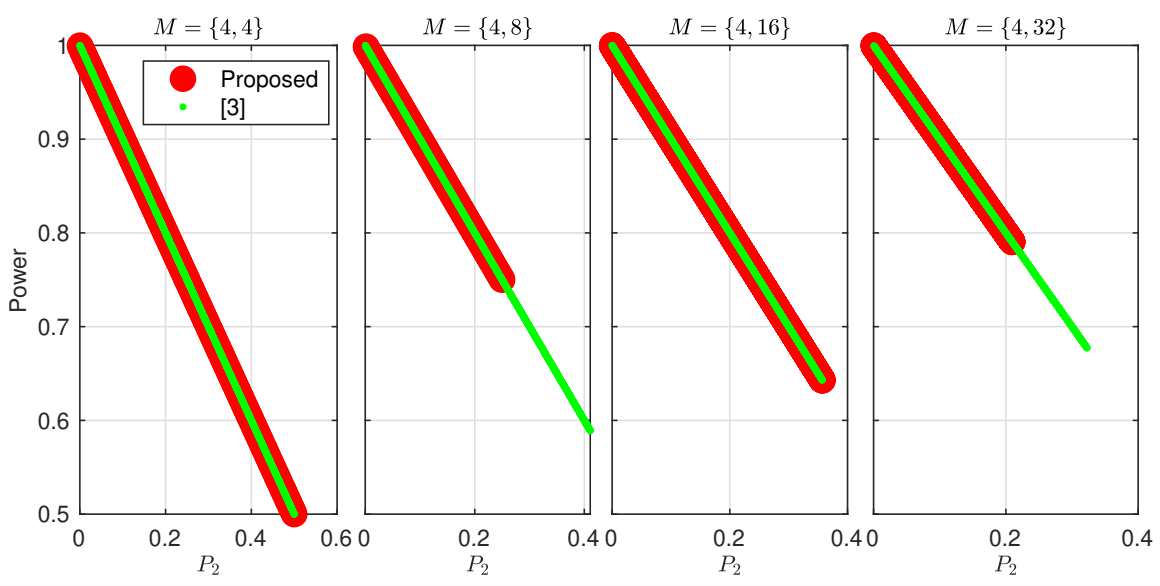

Figure 5: ${ }^{\left[R_{2,4}\right]\left[R_{3,5}\right]\left[R_{3,6}\right]}$ Comparison between the proposed system and [3].

\section{NuMERICAL RESUlTS}

This section presents the power ranges for some special cases of interest.

\section{A. $N=3$ and $M=4$}

In the case that $N=3$ and $M_{i}=4 \forall i$, the conditions become

$$
\begin{aligned}
& \beta_{3}>0 \\
& \beta_{2}>\beta_{3} \\
& \beta_{1}>\beta_{2}+\beta_{3} .
\end{aligned}
$$

Solving for $\beta_{1}, \beta_{2}$, and $\beta_{3}$, gives

$$
\begin{aligned}
& \beta_{1}=2 \beta_{3}+c+d \\
& \beta_{2}=\beta_{3}+c \\
& \beta_{3}=\left(\sqrt{6-3 c^{2}-2 d}-3 c-2 d\right) / 6
\end{aligned}
$$

with $0<c<1 / \sqrt{2}$ and $0<d<\sqrt{1-c^{2}}-c$. This result perfectly fits the numerical representation of the space of possible solutions as shown in Fig. $4 \mathrm{a}$, where the $x$-axis represents the value of $P_{3}$ and the $y$-axis shows the possible values of $P_{1}$ and $P_{2}$. The figure also shows the optimal power values, white circles, reported in [4] for different channel conditions. As can be noted from the figure, the optimal power values fall within the derived power ranges. Fig. $4 \mathrm{~b}$ shows that increasing the modulation order for the users reduces the power range significantly. Therefore, the derived ranges can be used to reduce the complexity of finding the optimal power allocation using brute force techniques. It is also worth noting that increasing $P_{3}$ reduces the ranges of $P_{1}$ and $P_{2}$. Therefore, in the case that $U_{3}$ has low signal-to-noise ratio (SNR) it will reduce the range of values that other users can choose from to optimize their performance.

\section{B. Other Typical Results}

The case of $N=2$ is widely used in the literature, and hence, we present the power ranges for selected modulation orders where $M_{1}=4$ and $M_{2} \in\{4,8,16,32\}$. In this case, the conditions are

$$
\beta_{1}>\beta_{2} \frac{\Delta_{1}}{\Delta_{2}} \max \left\{\left[2 D_{x}^{(2)}-1\right],\left[2 D_{y}^{(2)}-1\right]\right\}
$$

$\left[R_{2,4}\right]\left[R_{3,6}\right]$ Fig. 5 shows a comparison of the power allocation ranges produced by our proposed system to those produced by [3] for different square and rectangular QAM scenarios. Specifically for the cases where $M_{1}=4$ and $M_{2} \in$ $\{4,8,16,32\}$. As expected, for square QAM, $M_{2} \in\{4,16\}$, the power ranges are exactly the same. For rectangular QAM, $M_{2} \in\{8,32\}$, the ranges produced by [3] are larger as shown in the figure where the green line exceeds the red line. In those cases, even if the conditions proposed in [3] are satisfied, there will be overlap where some constellation points cross some decision boundaries.

Figs. 6 and 7 depict the ranges of possible power allocations when $N=4$ for various values of $M_{i}$. The $x$-axis represents the value of $P_{4}$ and the $y$-axis shows the possible values of $P_{1}$, $P_{2}$ and $P_{3}$. As can be noted from the figure, the power ranges for $P_{1}, P_{2}$ and $P_{3}$ are generally very narrow for high values of $P_{4}$, particularly when $M_{i}>4$ for any $i$. Consequently, the knowledge of these ranges will enable the system designer to search for particular power assignment values using extremely small search step-size without significantly increasing the time and computational complexity.

\section{CONCLUSION}

This work presented a derivation of the power ranges that can be assigned for NOMA systems with arbitrary number of users each of which uses a rectangular QAM with arbitrary modulation order. The power ranges obtained are presented in simple closed-form expressions, and all the work reported in the existing literature can be considered as special cases of the results presented in this work. The obtained expressions are invaluable for power optimizations problems where the search space for the optimum power can be reduced significantly, particularly when assigning the nearest user a high power. Similarly, increasing the number of users or the modulation 


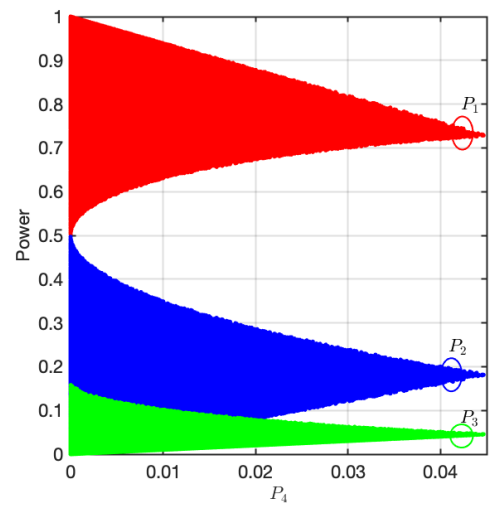

(a) $M=\{4,4,4,4\}$

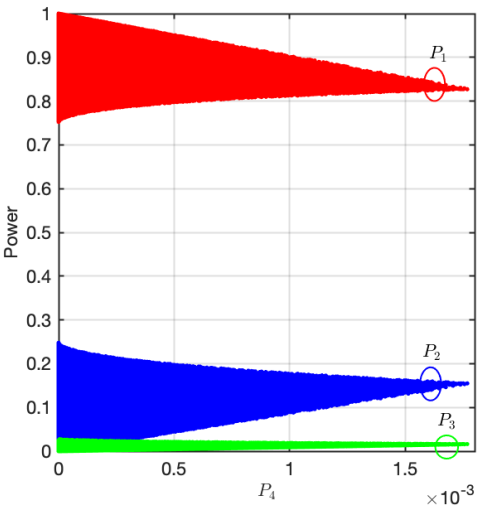

(b) $M=\{4,8,16,16\}$

Figure 6: Space of possible power allocation for $N=4$ and different values of $M$.

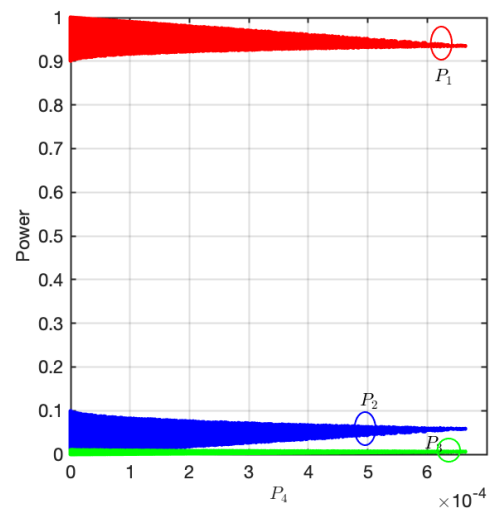

(a) $M=\{8,8,16,16\}$

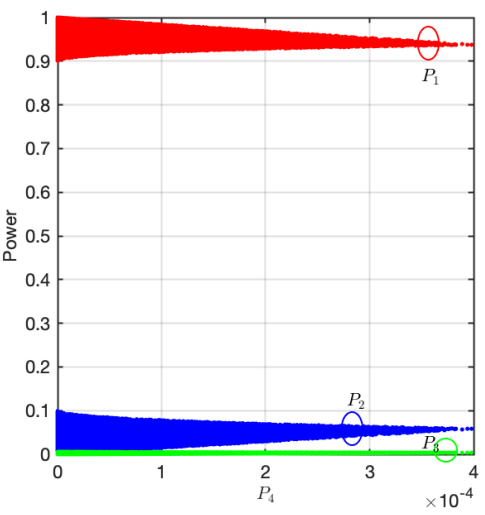

(b) $M=\{16,16,16,16\}$

Figure 7: Space of possible power allocation for $N=4$ and different values of $M$.

orders reduces the possible power assignment to a very narrow range of values.

\section{REFERENCES}

[1] T. Assaf, A. Al-Dweik, M. Moursi, H. Zeineldin, and M. Al-Jarrah, "NOMA receiver design for delay-sensitive systems," IEEE Syst. J., IEEE Xplore early access, 2020, doi=10.1109/JSYST.2020.3032878.

[2] — - "Exact bit error-rate analysis of two-user NOMA using QAM with arbitrary modulation orders," IEEE Commun. Lett., vol. 24, no. 12, pp. 2705-2709, 2020

[3] F. KARA and H. KAYA, "A True Power Allocation Constraint for Non-Orthogonal Multiple Access with M-QAM Signalling," in 2020 IEEE Microwave Theory and Techniques in Wireless Communications (MTTW), vol. 1, 2020, pp. 7-12.

[4] T. Assaf, A. Al-Dweik, M. Moursi, and H. Zeineldin, "Exact BER performance analysis for downlink NOMA systems over Nakagami- $m$ fading channels," IEEE Access, vol. 7, pp. 134 539-134 555, 2019.

[5] G. Li, D. Mishra, and H. Jiang, "Channel-aware power allocation and decoding order in overlay cognitive NOMA networks," IEEE Trans. Veh. Technol., vol. 69, no. 6, pp. 6511-6524, 2020.

[6] Y. Fu, L. Salaün, C. W. Sung, and C. S. Chen, "Subcarrier and power allocation for the downlink of multicarrier NOMA systems," IEEE Trans. Veh. Technol., vol. 67, no. 12, pp. 11 833-11 847, 2018.

[7] M. R. Zamani, M. Eslami, M. Khorramizadeh, and Z. Ding, "Energyefficient power allocation for NOMA with imperfect CSI," IEEE Trans. Veh. Technol., vol. 68, no. 1, pp. 1009-1013, 2019.

[8] J. A. Oviedo and H. R. Sadjadpour, "A fair power allocation approach to NOMA in multiuser SISO systems," IEEE Trans. Veh. Technol., vol. 66 , no. 9, pp. 7974-7985, 2017.
[9] L. Bariah, S. Muhaidat, and A. Al-Dweik, "Error probability analysis of non-orthogonal multiple access over Nakagami- $m$ fading channels," IEEE Trans. Commun., vol. 67, no. 2, pp. 1586-1599, 2019.

[10] ${ }^{\left[R_{1,2}\right]}$ X. Zhang, L. Yang, Z. Ding, J. Song, Y. Zhai, and D. Zhang, "Sparse Vector Coding-Based Multi-Carrier NOMA for In-Home Health Networks," IEEE J. Sel. Areas Commun., vol. 39, no. 2, pp. 325-337, 2021.

[11] E. M. Almohimmah and M. T. Alresheedi, "Error analysis of NOMAbased vlc systems with higher order modulation schemes," IEEE Access, vol. 8, pp. 2792-2803, 2020.

[12] I. Lee and J. Kim, "Average symbol error rate analysis for nonorthogonal multiple access with $m$-ary QAM signals in rayleigh fading channels," IEEE Commun. Lett., vol. 23, no. 8, pp. 1328-1331, 2019.

[13] M. Aldababsa et al., "Bit error rate for NOMA network," IEEE Commun. Lett., vol. 24, no. 6, pp. 1188-1191, 2020.

[14] ${ }^{\left[R_{2,2}\right]}$ P. Singya et al., "A Survey on Higher-Order QAM Constellations: Technical Challenges, Recent Advances, and Future Trends," IEEE Open Journal of the Communications Society, vol. 2, pp. 617-655, 2021.

[15] $\left[R_{2,2}\right]$ R. Prodan, B. Shen, and A. Kliger, "Constellation mapping for EPoC LDPC,” Nov. 2013, accessed April 23 2021. [Online]. Available: https://www.ieee802.org/3/bn/public/nov13/prodan_3bn_02_1113.pdf

[16] ${ }^{\left[R_{2,2}\right]}$ F. Sun et al., "All-optical rectangular-QAM regenerators based on phase-preserving I/Q shaping," Optics Commun., vol. 467, p. 125634 , 2020. 